\title{
Adaptive Singular Value QRST Cancellation for the Analysis of Short Single Lead Atrial Fibrillation Electrocardiograms
}

\author{
R Alcaraz ${ }^{1}$, JJ Rieta $^{2}$ \\ ${ }^{1}$ Innovation in Bioengineering Research Group. University of Castilla-La Mancha, Cuenca, Spain \\ ${ }^{2}$ Biomedical Synergy. Valencia University of Technology, Valencia, Spain
}

\begin{abstract}
Atrial fibrillation $(A F)$ is the most commonly diagnosed sustained supraventricular arrhythmia in clinical practice and is characterized by uncoordinated atrial activation. The proper analysis and characterization of $A F$ from surface ECG recordings requires to cancel out ventricular activity (i.e. QRS-T complexes). Some powerful methods exploit the spatial diversity of multi-lead ECG, however, their performance is seriously reduced in single-lead environments. For this latter case, techniques based on averaged beat subtraction (ABS) are the most widely used. However, these methods are very sensitive to $Q R S-T$ wave variations, thus, a high quality QRS-T cancellation template may be difficult to obtain when only short length recordings are available. To overcome these difficulties, a new QRS-T cancellation method based on singular value decomposition (SVD) of each single beat is presented. This methodology was tested and validated using a significative database with simulated and real $A F$ recordings. The results showed that SVD is able to obtain a very accurate ventricular activity (VA) representation, thus providing high quality atrial activity (AA) extraction in short and single-lead $A F$ recordings. Therefore, the inherent limitations in ABS techniques due to variations in the QRS-T shape could be avoided.
\end{abstract}

\section{Introduction}

Atrial fibrillation (AF) is the most commonly diagnosed sustained arrhythmia in clinical practice and affects up to $1 \%$ of the general population [1]. Considering its prevalence with age, this arrhythmia affects up to $15 \%$ of the population older than 80 and has an incidence that doubles with each advancing decade [2,3]. During AF, the coordinated activation of the atria is replaced by an uncoordinated atrial activation that leads to the deterioration of atrial mechanical function with a consequent increased risk of stroke and mortality[2].

The surface ECG provides a noninvasive way to study
AF mechanisms and to investigate the effects of remodelling and treatment on AF [4]. Some of the advantages of using the surface ECG include the ability to record data for a long period of time, the minimal cost and risks involved compared with invasive electrophysiological studies, and its reflection of the global activity in the atria and ventriculus during AF [4]. However, the proper analysis and characterization of $\mathrm{AF}$ from $\mathrm{ECG}$ recordings requires the extraction or cancellation of the signal components associated with ventricular activity (VA), that is, the QRS complex and the T-wave (QRS-T)[5]. Unfortunately, a number of facts hinder this operation. First, atrial activity (AA) presents in the surface ECG much lower amplitude than its ventricular counterpart. Additionally, both phenomena possess spectral distributions that notably overlap, rendering linear filtering solutions unsuccessful [6].

To date, several ways to extract the AA from surface ECG recordings have been presented. The most powerful techniques are those that exploit the spatial diversity of the multilead ECG [7, 8], such as the method that solves the blind source separation problem [6]. However, the performance of these techniques is seriously reduced when they analyze the early stages of AF, i. e., paroxysmal AF, because the recordings are usually obtained from a Holter system with no more than two or three electrodes. Such a reduced number of leads is not sufficient to exploit the spatial information of the ECG, rendering those techniques based on averaged beat subtraction (ABS) the main alternatives. However, these techniques are very sensitive to QRST wave variations, and the estimated AA can be affected by some QRS-T residues, which may be important owing to the low AA amplitude [9]. Additionally, in clinical practice the ECG consists of 10 seconds in length recordings and, therefore, high quality QRS-T cancellation template may be difficult to obtain [10].

Thereby, this work presents a new cancellation method based on singular value decomposition (SVD) of each single beat capable to cancel out the QRS-T complexes in short duration and single-lead AF electrocardiograms. Thus, the proposed SVD-based methodology tries to ex- 
tract the basis signal corresponding to the ventricular activity by exploiting the mutual information contained on each ECG beat.

\section{Materials}

\subsection{Database}

The presented methodology was validated using a database composed of twenty 15 seconds-length simulated AF recordings and twenty 15 seconds-length AF ECGs. Simulated recordings allowed to compare the estimated and original AA, as it was known a priori. Synthesized AF signals were created from the combination of AA and VA, which were synthesized separately. The AA was generated from the smooth concatenation of successive TQ segments extracted from AF ECGs. The VA was synthesized from normal sinus rhythm ECGs, after P-wave cancellation [9].

In addition, to evaluate the suitability of the algorithm to be applied over real scenarios, which was the final purpose, real AF recordings were used. These signals were obtained from Holter systems of two leads (V1 and II), digitized at a sampling frequency of $128 \mathrm{~Hz}$ and a resolution of 12 bits. Lead V1 was the input signal of the AA estimation approach, as it was the signal with higher AA content.

\subsection{Data preprocessing}

The real ECG recordings were preprocessed in order to reduce noise, nuisance interferences and improve later analysis. Firstly, baseline wander was removed making use of bidirectional high pass filtering with $0.5 \mathrm{~Hz}$ cutt-off frequency [11]. Secondly, high frequency noise was reduced with an eight order bidirectional IIR Chebyshev low pass filtering, whose cut-off frequency was $70 \mathrm{~Hz}$ [12]. Finally, powerline interference was removed through adaptive notch filtering, which preserves the ECG spectral information [13]. Moreover, these signals were upsampled to $1 \mathrm{kHz}$ in order to improve time alignment accuracy for QRS-T complex subtraction.

\section{Methods}

\subsection{AA extraction algorithm}

The ECG signal presents a high degree of temporal redundancy which could be exploited in order to cancel VA. Indeed, the QRS-T waveform usually exhibits a recurrent pattern, although different QRS-T morphologies as well as minor variations in the QRS-T waveform may occur [9]. Thus, all $\mathrm{R}$ waves were firstly detected making use of the Pan \& Tompkins technique [14]. Next, the $i$-th QRS-T complex start point was defined as $s_{i}=r_{i}-0.3 \cdot R R_{m i n}$, being $r_{i}$ the $\mathrm{R}$ peak wave event and $R R_{\text {min }}$ the minimum $\mathrm{R}-\mathrm{R}$ interval found in the ECG. The $i$-th QRS-T complex end point was selected as $e_{i}=r_{i}+0.7 \cdot R R_{\min }$ [15]. Each QRS-T complex was assumed to be represented by a column vector of the matrix $\mathbf{X} \in \Re^{\mathbf{L} \times \mathbf{N}}$ :

$$
\mathbf{X}=\left[\mathbf{x}_{1}, \mathbf{x}_{2}, \ldots, \mathbf{x}_{\mathbf{N}}\right]
$$

where $\mathbf{x}_{\mathbf{i}}$ contains $L$ samples of $i$-th complex, and $N$ is the complex number in the analyzed ECG. Note that all beats were temporally aligned using its $\mathrm{R}$ peak timing.

The Singular Value Decomposition (SVD) of the matrix $\mathrm{X}$ can be expressed as:

$$
\mathbf{X}=\mathbf{U S V}^{\mathbf{T}}
$$

where $\mathbf{U} \in \Re^{\mathbf{L} \times \mathbf{N}}$ is an unitary matrix so that $\mathbf{U U}^{\mathbf{T}}=\mathbf{I}$, $\mathbf{S} \in \Re^{\mathbf{N} \times \mathbf{N}}$ is an diagonal matrix, and $\mathbf{V} \in \Re^{\mathbf{N} \times \mathbf{N}}$ fulfills $\mathbf{V V}^{\mathbf{T}}=\mathbf{I}$. The matrix $\mathbf{U}$ contains the $N$ normalized principal components of $\mathbf{X}$, so that the columns of $\mathbf{U}=\left[\mathbf{u}_{\mathbf{1}} \ldots, \mathbf{u}_{\mathbf{N}}\right]$ are the eigenvectors of $\mathbf{X}$, and their cross-correlations are nulls. The matrix $\mathbf{S}$ contains the amplitude coefficients corresponding to the $N$ principal components of $\mathbf{X}$. These coefficients are called eigenvalues or singular values and are sorted in descending order. Thus, the $N$ non-normalized principal components can be obtained as the columns of the matrix $\mathbf{P}=\mathbf{U S}$, and can be interpreted as follows:

- The most significant component is related to the main QRS-T waveform.

- Subsequently, there are several components related to AA.

- The remaining components correspond to noise.

Atrial activity can be viewed as being uncoupled to VA. Thus, each observed beat can be modelled as a sum of atrial activity $\left(\mathbf{X}_{\mathbf{A A}}\right)$ and ventricular activity ( $\left.\mathbf{X}_{\mathbf{V A}}\right)$ [7]:

$$
\mathbf{X}=\mathbf{X}_{\mathbf{A A}}+\mathbf{X}_{\mathbf{A V}}
$$

Hence, the first principal component, which is called $\mathbf{t}$, can be used as QRS-T template to cancel out VA. However, since considerable $\mathrm{R}$ peak amplitude differences between each individual QRS-T complex and template were found, t amplitude was individually adapted to each beat:

$$
\mathbf{t}_{\mathbf{i}}=\frac{\mathbf{Q R}_{\mathbf{i}}}{\mathbf{Q R}} \cdot \mathbf{t}
$$

where $\mathbf{Q} \mathbf{R}_{\mathbf{i}}$ and $\mathbf{Q} \mathbf{R}_{\mathbf{t}}$ are the distances between the $Q$ and $R$ points of the $i$-th complex and template, respectively. Thus, the AA estimation $\left(\widehat{\mathbf{X}}_{\mathbf{A A}}\right)$ was obtained as:

$$
\widehat{\mathbf{X}}_{\mathbf{A A}}=\mathbf{X}-\mathbf{T}
$$

being $\mathbf{T}$ the matrix constituted by the column vectors $\mathbf{t}_{\mathbf{i}}$ :

$$
\mathbf{T}=\left[\mathbf{t}_{\mathbf{1}}, \mathbf{t}_{\mathbf{2}}, \ldots, \mathbf{t}_{\mathbf{N}}\right]
$$




\subsection{Performance assessment}

The AA estimation performance in simulated recordings was computed by comparing the estimated and original AA in terms of Pearson correlation index. This coefficient measures the similarities between two signals, and becomes 1 in the case of perfect matching and 0 in the case of completely different and non-dependent signals.

An additional performance parameter was also introduced to evaluate AA estimation in real recordings. Thus, ventricular activity reduction (VAR) was defined as the ratio between the spectral power concentrations of the original ECG and obtained AA in $0.5-2.5 \mathrm{~Hz}$ band:

$$
V A R=\sum_{f=0.5 H z}^{2.5 H z} \frac{P_{x}(f)}{P_{\widehat{x}_{A A}}(f)}
$$

where $P_{x}$ and $P_{\widehat{x}_{A A}}(f)$ are the ECG and estimated AA power spectrums, respectively.

\section{Results}

The proposed methodology was firstly applied to the simulated AF signals. The Pearson correlation index obtained for each recording is detailed in Table 1 , being 0.92 \pm 0.07 in average. The estimated AA quality is illustrated in Figure 1, which shows the ECG, original and estimated AA corresponding to a typical recordings (P8). As can be appreciated, the estimated AA nearly matches the original AA, without any QRS-T residue.

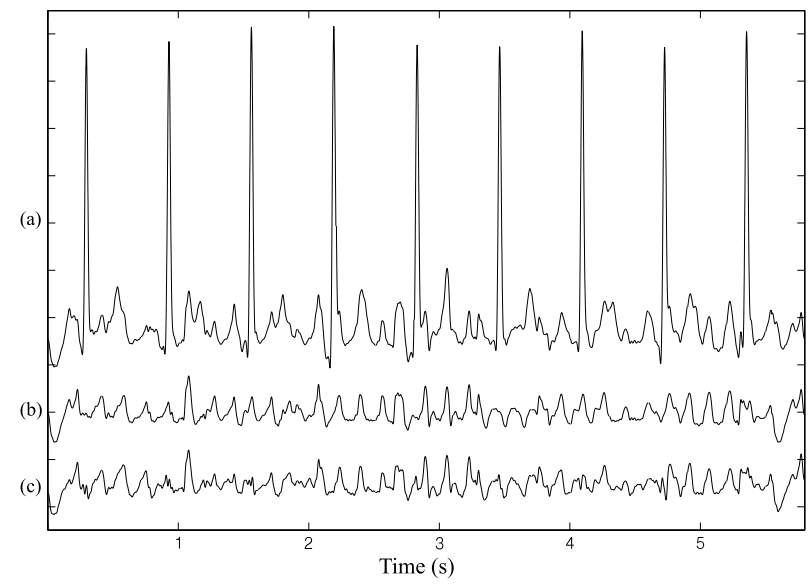

Figure 1. (a) Simulated 6 seconds-length ECG signal. (b) Real AA. (c) Estimated AA.

Secondly, the same methodology was applied to the recordings obtained from real AF patients. In this case, the VAR parameter was used to evaluate the proposed method performance. The VAR value obtained for each patient is shown in Table 2, being $12.14 \pm 1.99$ in average. Taking into account that AA presents much less amplitude than VA, these results indicate that the proposed approach is able to estimate AA free from QRS-T residua. Analyzing real $\mathrm{AF}$ recordings, the AA extraction quality is illustrated in Figure 2. Again, it can be observed that AA can be extracted without QRS-T residues.

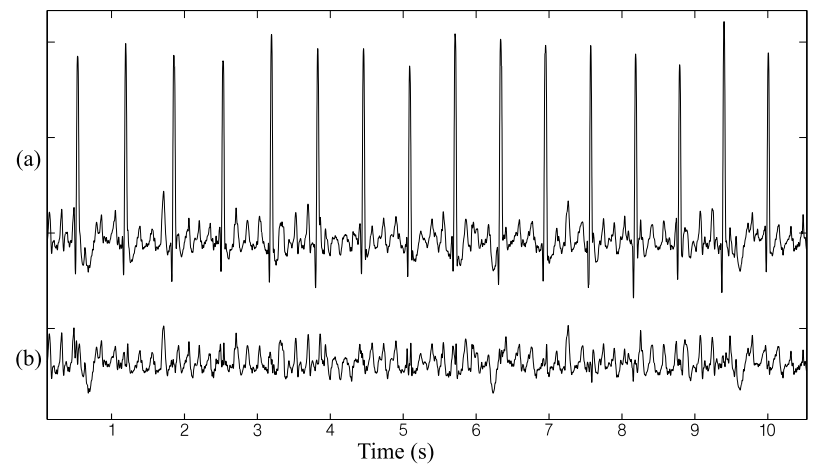

Figure 2. (a) Real 11 seconds-length ECG signal. (b) Estimated AA.

\section{Discussion and conclusions}

The AA estimation in short AF episodes requires the implementation of QRS-T cancellation techniques for singlelead ECGs, rendering ABS techniques the unique existing solutions. In this work, an alternative method based on SVD concepts has been presented. The proposed methodology tries to overcome the limitations of the ABS strategies, which are highly sensitive to QRS-T morphology variations.

The results have shown that SVD is able to obtain a very accurate representation of VA, thus providing high quality AA extraction in short and single-lead AF recordings. Thereby, it can be concluded that a high accuracy QRS-T template is obtained, since dynamics in the QRS-T waveform are considered. Thus, the inherent limitations in ABS techniques could be avoided.

To date, other two methods have been proposed to extract AA from short duration and single-lead AF recordings. Thus, Castells et. al. [9] presented a new method based on PCA concepts, however, the correlation index between estimated and original AA was $0.774 \pm 0.106$ in average. Recently, Lemay et. al. [10] has presented other method, in which cancellation during the JQ intervals is carried out using dominant $\mathrm{T}$ and $\mathrm{U}$ wave methodology and AA estimation during the QRS intervals is performed making use of a weighted sum of sinusoids. Because of the estimated and real AA during the QRS interval could be very different when AA is very irregular, it can be considered that this new proposed method can obtain a more accurate AA estimation. 
Table 1. Correlation values obtained for the simulated AF ECGs.

\begin{tabular}{cccccccccc} 
S1 & S2 & S3 & S4 & S5 & S6 & S7 & S8 & S9 & S10 \\
\hline 0.91 & 0.87 & 0.91 & 0.88 & 0.95 & 0.89 & 0.87 & 0.91 & 0.89 & 0.84 \\
\hline \hline S11 & S12 & S13 & S14 & S15 & S16 & S17 & S18 & S19 & S20 \\
\hline 0.82 & 0.84 & 0.93 & 0.94 & 0.90 & 0.88 & 0.80 & 0.82 & 0.94 & 0.92 \\
\hline \hline
\end{tabular}

Table 2. VAR values of the estimated AA from real AF recordings.

\begin{tabular}{cccccccccc}
$\mathbf{R 1}$ & $\mathbf{R 2}$ & $\mathbf{R 3}$ & $\mathbf{R 4}$ & $\mathbf{R 5}$ & $\mathbf{R 6}$ & $\mathbf{R 7}$ & $\mathbf{R 8}$ & $\mathbf{R 9}$ & $\mathbf{R 1 0}$ \\
\hline 12.58 & 9.32 & 13.42 & 15.24 & 10.61 & 13.71 & 14.50 & 8.81 & 9.11 & 13.14 \\
\hline \hline R11 & $\mathbf{R 1 2}$ & $\mathbf{R 1 3}$ & $\mathbf{R 1 4}$ & $\mathbf{R 1 5}$ & $\mathbf{R 1 6}$ & $\mathbf{R 1 7}$ & $\mathbf{R 1 8}$ & $\mathbf{R 1 9}$ & $\mathbf{R 2 0}$ \\
\hline 11.20 & 13.38 & 13.63 & 13.42 & 14.58 & 13.33 & 14.38 & 9.59 & 11.96 & 11.68 \\
\hline \hline
\end{tabular}

\section{Acknowledgements}

This work was partly supported by the projects 20070086 from the R+D+i Vice-rectorate of the Valencia University of Technology, GV06/299 from Consellería de Empresa, Universidad y Ciencia de la Generalitat Valenciana and TEC2007-64884 from the Spanish Ministry of Education and Science.

\section{References}

[1] Kannel WB, Abbott RD, Savage DD, McNamara PM. Epidemiologic features of chronic atrial fibrillation: the Framingham study. N Engl J Med Apr 1982;306(17):1018-1022.

[2] Fuster V, Rydén LE, Cannom DS, et. al. ACC/AHA/ESC 2006 guidelines for the management of patients with atrial fibrillation: a report of the American College of Cardiology/American Heart Association task force on practice guidelines and the european society of cardiology committee for practice guidelines (writing committee to revise the 2001 guidelines for the management of patients with atrial fibrillation): developed in collaboration with the european heart rhythm association and the heart rhythm society. Circulation Aug 2006;114(7):e257-e354.

[3] Furberg CD, Psaty BM, Manolio TA, Gardin JM, Smith VE, Rautaharju PM. Prevalence of atrial fibrillation in elderly subjects (the Cardiovascular Health Study). Am J Cardiol Aug 1994;74(3):236-241.

[4] Petrutiu S, Ng J, Nijm GM, Al-Angari H, Swiryn S, Sahakian AV. Atrial fibrillation and waveform characterization. A time domain perspective in the surface ECG. IEEE Eng Med Biol Mag 2006;25(6):24-30.

[5] Slocum J, Sahakian A, Swiryn S. Diagnosis of atrial fibrillation from surface electrocardiograms based on computerdetected atrial activity. J Electrocardiol Jan 1992;25(1):1-8.

[6] Rieta JJ, Castells F, Sánchez C, Zarzoso V, Millet J. Atrial activity extraction for atrial fibrillation analysis using blind source separation. IEEE Trans Biomed Eng Jul 2004; 51(7):1176-1186.
[7] Stridh M, Sörnmo L. Spatiotemporal QRST cancellation techniques for analysis of atrial fibrillation. IEEE Trans Biomed Eng Jan 2001;48(1):105-111.

[8] Langley P, Rieta JJ, Stridh M, Millet J, Sörnmo L, Murray A. Comparison of atrial signal extraction algorithms in 12lead ECGs with atrial fibrillation. IEEE Trans Biomed Eng Feb 2006;53(2):343-346.

[9] Castells F, Mora C, Rieta JJ, Moratal-Pérez D, Millet J. Estimation of atrial fibrillatory wave from single-lead atrial fibrillation electrocardiograms using principal component analysis concepts. Med Biol Eng Comput Sep 2005; 43(5):557-560.

[10] Lemay M, Vesin JM, van Oosterom A, Jacquemet V, Kappenberger L. Cancellation of ventricular activity in the ecg: evaluation of novel and existing methods. IEEE Trans Biomed Eng Mar 2007;54(3):542-546.

[11] Dotsinsky I, Stoyanov T. Optimization of bi-directional digital filtering for drift suppression in electrocardiogram signals. J Med Eng Technol 2004;28(4):178-180.

[12] Sun Y, Chan K, Krishnan SM. ECG signal conditioning by morphological filtering. Comput Biol Med Nov 2002; 32(6):465-479.

[13] Ferdjallah M, Barr RE. Adaptive digital notch filter design on the unit circle for the removal of powerline noise from biomedical signals. IEEE Trans Biomed Eng Jun 1994; 41(6):529-536.

[14] Pan J, Tompkins WJ. A real-time QRS detection algorithm. IEEE Trans Biomed Eng Mar 1985;32(3):230-236.

[15] Shkurovich S, Sahakian AV, Swiryn S. Detection of atrial activity from high-voltage leads of implantable ventricular defibrillators using a cancellation technique. IEEE Trans Biomed Eng Feb 1998;45(2):229-234.

Address for correspondence:

Raúl Alcaraz Martínez

E. U. Politécnica de Cuenca

Campus Universitario

16071 Cuenca (Spain)

raul.alcaraz@uclm.es 